\title{
Análise comparativa de lajes de pontes em balanço com base no método de elementos
}

\section{finitos}

\author{
Comparative analysis of cantilever bridge slab thickness based on the finite element method
}

Análisis comparativo de los espesores de las losas de los puentes en voladizo basado en el método de

los elementos finitos

Recebido: 27/09/2021 | Revisado: 05/10/2021 | Aceito: 10/11/2021 | Publicado: 14/11/2021

\author{
Luan Bastos Correia \\ ORCID: https://orcid.org/0000-0003-0660-8845 \\ Universidade Federal Fluminense, Brasil \\ E-mail: luanbastos@id.uff.br \\ Luiz Carlos Mendes \\ ORCID: https://orcid.org/0000-0003-1747-5179 \\ Universidade Federal Fluminense, Brasil \\ E-mail: 1carlos@predialnet.com.br
}

\begin{abstract}
Resumo
O processo de construção de pontes rodoviárias de concreto armado exige estudo e análise da ação de cargas permanentes e variáveis, além de outros fatores como as ações dinâmicas oriundas da movimentação e tráfego de veículos sobre o tabuleiro. A propagação do capitalismo, associada ao fenômeno de globalização ocasiono o crescimento exponencial do fluxo de veículos, consequentemente maiores solicitações estruturais, esses fatores apresentam grande relevância no planejamento e desenvolvimento no projeto de construção de superestruturas, pois estão inerentes ao surgimento de momentos transversais despertados na direção longitudinal das lajes de pontes em balanço. A busca pela análise correta e consideração desses aspectos para avaliação estrutural de uma ponte motivou o desenvolvimento desse estudo, para melhor compreensão dos esforços despertados no elemento estrutural, bem como a solução eficiente para o mesmo. A análise da variação de espessuras de lajes de pontes em balanço é um fator preponderante no embasamento dessa pesquisa. O estudo abordou o modelo matemático semi-analítico desenvolvido por Bakht e Jaeger (1985) e comparado com os resultados obtidos por Robalo (2013) por meio do software baseado no método de elementos finitos, foi realizada uma comparação e análise dos momentos fletores despertados na laje em balanço, correlacionando aos fatores relacionados ao surgimento desses esforços. A pesquisa utilizou o programa Microsoft Excel e os resultados obtidos por Robalo (2013) com o software SAP2000. Conclui-se nesse trabalho a proporcionalidade da variação de espessuras de lajes com a intensidade de momentos de picos e o surgimento de fissuras na peça estrutural.
\end{abstract}

Palavras-chave: Laje em balanço; Carga; Espessura; Momentos transversais; Pontes.

\begin{abstract}
The construction process of reinforced concrete highway bridges requires study and analysis of the action of permanent and variable loads, in addition to other factors such as the dynamic actions arising from the movement and traffic of vehicles on the deck. The spread of capitalism, associated with the phenomenon of globalization causes the exponential growth of vehicle flow, consequently greater structural demands, these factors have great relevance in planning and development in the design of superstructure construction, because they are inherent to the emergence of transverse moments aroused in the longitudinal direction of the cantilever bridge slabs. The search for the correct analysis and consideration of these aspects for the structural evaluation of a bridge motivated the development of this study, for a better understanding of the efforts aroused in the structural element, as well as the efficient solution for it. The analysis of the variation in the thickness of cantilever bridge slabs is a preponderant factor in the foundation of this research. The study approached the semi-analytical mathematical model developed by Bakht and Jaeger (1985) and compared with the results obtained by Robalo (2013) by means of software based on the finite element method, a comparison and analysis of bending moments aroused in the cantilever slab was performed, correlating the factors related to the emergence of these efforts. The research used the Microsoft Excel program and the results obtained by Robalo (2013) with the SAP2000 software. It is concluded in this work the proportionality of the variation of slab thickness with the intensity of peak moments and the appearance of cracks in the structural part.
\end{abstract}

Keywords: Deck slab overhangs; Load; Thickness; Transverse moments; Bridge. 


\begin{abstract}
Resumen
El proceso de construcción de puentes de carretera de hormigón armado requiere el estudio y análisis de la acción de las cargas permanentes y variables, además de otros factores como las acciones dinámicas derivadas del movimiento y tráfico de vehículos sobre el tablero. La propagacion del capitalismo, asociada al fenómeno de la globalización que provoca el crecimiento exponencial del flujo de vehículos, y en consecuencia las mayores demandas de estructura, estos factores presentan una gran relevancia en la planificación y desarrollo del proyecto de construcción de superestruturas, ya que son inerentes al surgimiento de momentos transversales despertados en la dirección longitudinal de los ejes de ponte en equilibrio. La búsqueda de un análisis correcto y la consideración de estos aspectos para la evaluación estructural de un puente motivó el desarrollo de este estudio, para una mejor comprensión de los esfuerzos realizados en el elemento estructural, así como la solución eficiente para el mismo. El análisis de la variación del espesor de las losas de los puentes en voladizo es un factor preponderante en la fundamentación de esta investigación. El estudio aborda el modelo matemático semianalítico desarrollado por Bakht y Jaeger (1985) y comparado con los resultados obtenidos por Robalo (2013) mediante el software basado en el método de elementos finitos, se realizó una comparación y análisis de los momentos de los factores despertados en el juego en equilibrio, correlacionando los factores relacionados con el surgimiento de estos esfuerzos. La investigación utilizó el programa Microsoft Excel y los resultados obtenidos por Robalo (2013) con el software SAP2000. Se concluye en este trabajo la proporcionalidad de la variación de espesores de láminas con la intensidad de los momentos de picos y el surgimiento de fisuras en la pieza estructural.
\end{abstract}

Palabras clave: Losa en voladizo; Cargar; Espesor; Momentos transversales; Puentes.

\title{
1. Introdução
}

Pinho (2007) explica que desde a ambiguidade ao longo do processo de formação e agrupamento da população em comunidades (aldeias e cidades), começou-se a surgir as preocupações dos povos para vencerem obstáculos que dificultariam sua evolução e desenvolvimento, e então surgem as pontes.

Pfeil (1979) define pontes como construções destinadas a transposição de obstáculos à continuidade do leito normal de uma via, podendo ser obstáculos naturais como leitos de rios, vales profundos, braços de mar ou obstáculos criados pelo homem com o desenvolvimento e crescimentos dos grandes centros urbanos, sendo esse último caso chamado de viadutos.

Marchetti (2018) sintetiza o conceito de pontes como sendo obras destinadas a vencer obstáculos e permitir a continuidade de vias de comunicação. A utilização do termo ponte é empregada quando o obstáculo transposto é um rio, e viaduto quando o obstáculo a ser vencido é um vale ou via.

Os principais materiais utilizados na construção dessas estruturas, também conhecidas como obras de artes especiais (OAE's), foram a pedra e posteriormente a madeira, onde é possível identificar grande contribuição dos povos romanos na construção de pontes de pedra e que resistem até os dias atuais (Leonhardt, 1979).

De modo geral diversos engenheiros projetistas definem alguns critérios preponderantes para a concepção de ponte, tais como:

- Visualização do caminhamento das cargas, desde a aplicação até o ponto de dissipação na fundação;

- $\quad$ Projetar fundações sob cargas a suportar;

- $\quad$ Princípio do caminho mais curto e eficiente de dissipação das cargas;

- $\quad$ Princípio da rigidez;

- $\quad$ Princípio da distribuição, entre outros.

No projeto de uma ponte deve-se levar em consideração também a ação dos carregamentos externos, bem como os carregamentos provenientes da própria estrutura. As ações são definidas como causas que provocam deformações ou esforços nas estruturas. Moderadamente dividem-se em ações permanentes, variáveis e excepcionais.

As ações permanentes são aquelas que podem ser consideradas constantes ao longo da vida da construção, são elas: peso próprio da estrutura, peso da pavimentação, dos trilhos, dormentes, empuxos de terra e líquido. As ações variáveis apresentam grande variação em torno de sua média ao longo da vida útil da construção, tais como: forças de aceleração e 
frenagem dos veículos cargas de vento, pressão da água em movimento efeito dinâmico da água, variações de temperaturas, entre outras.

As ações excepcionais são aquelas cujas ocorrências se dão em condições anormais podem ser exemplificadas como: explosões, choques de objetos móveis, fenômenos naturais pouco frequentes como ventos ou enchentes catastróficos, abalos sísmicos, entre outros.

O processo de globalização e desenvolvimento do mundo, associado ao capitalismo, estimulou o crescimento exponencial do fluxo e tráfego de pessoas, veículos e mercadorias pelas vias de acesso, tais como rodovias, pontes e viadutos, de forma que gerasse uma maior atenção por parte de profissionais responsáveis pelo planejamento e construção das vias de acesso, em especial as pontes destinadas a atenderem a alta demanda de solicitações de cargas sobre a susperestrutura com eficiência e segurança, dentro dos estados limites de utilização e serviço da estrutura.

A alta demanda de solicitações e tipos de ações sobre as pontes exigem estudos e análises minuciosos, entre eles as ações e comportamentos das peças estruturais das pontes, como os balanços da laje (cantilever), os quais são fundamentais no processo de dimensionamento de uma superestrutura, constituída pelos tabuleiros.

Bakht e Mufti (2015) conceituam o surgimento de momentos transversais de pico nas lajes em balanço devido à aplicação de cargas concentradas, os quais estão relacionados ao surgimento de fissuras na direção longitudinal e que acarretam a redução da rigidez flexional da peça. Além disso, os efeitos gerados pelos momentos transversais não se limitam a balanço, mas se estendem até o painel interno da laje da ponte.

Estimulado pela necessidade de contribuição acadêmica e científica em um estudo com pouca abordagem e aprofundamento no meio acadêmico, este trabalho almeja contribuir de forma científica através da análise estrutural de lajes de pontes em balanço, bem como os problemas encontrados com o surgimento de momentos transversais de pico que estão submetidos à estrutura durante a aplicação de cargas pontuais, e quem deve ser idealizadas e dimensionadas para suportar as solicitações dentro dos estados limites de serviço e utilização da construção.

O estudo apresentado foi fundamentado no modelo matemático de análise de lajes de pontes em balanço (cantilever) formulado e atualizado por Bakht e Mufti (2015), através da investigação dos esforços de momentos fletores transversais despertados na direção longitudinal do balanço das pontes, devido à aplicação de cargas concentradas e que ocorrem picos de intensidades de momentos em virtude da variação da razão de espessura do tabuleiro e o posicionamento das cargas concentradas ao longo da superfície em balanço.

Os resultados obtidos através do modelo semi-analítico foram comprados com os valores encontrados por Robalo (2013), onde se utilizou o software de análise estrutural SAP2000, programa baseado no método de elementos finitos.

Os principais objetivos do estudo são:

- Apresentar a análise do comportamento estrutural de lajes de pontes em balanço correlacionando com suas espessuras;

- Realizar um estudo comparativo dos resultados obtidos através da utilização de software de análise estrutural (SAP2000) por Robalo (2013) com os resultados do estudo semi-analítico proposto por Bakht e Mufti (2015);

- Avaliar o comportamento do elemento estrutural.

\section{Metodologia}

A pesquisa foi elaborada através de uma análise comparativa de um estudo semi-analítico, a partir do método qualitativo, o qual auxilia na melhor compreensão de fenômenos estudados individualmente.

A fundamentação teórica foi estruturada pelo embasamento das normas técnicas brasileiras NBR 7187 (ABNT, 2021) de Projeto de pontes, viadutos e passarelas de concreto e NBR 7188 (ABNT, 2013) de Carga móvel rodoviária e de pedestres 
em pontes, viadutos, passarelas e outras estruturas, além do conceito do método de análise de pontes em balanço (cantilever) formulado por Bakht e Jaeger (1985), sendo atualizado por Bakht e Mufti (2015).

Para elaboração do memorial de cálculos matemáticos utilizados no modelo semi-analítico apresentado, utilizou-se o software Microsoft Excel com intuito de dinamizar e facilitar o levantamento do conjunto de resultados para cada exemplo analisado.

Os resultados do modelo numérico utilizados neste trabalho para análise comparativa foram consultados a partir de Robalo (2013) e comparados com o modelo semi-analítico proposto por Bakht e Mufti (2015). Os dados de entrada aderidos nesse estudo semi-analítico foram apresentados no capítulo 5 deste trabalho.

A organização e compreensão deste estudo ocorrem através da análise comparativa dos resultados obtidos por Robalo (2013) com modelagem de lajes de pontes em balanço com auxílio do software SAP2000, programa baseado no Método de Elementos Finitos.

Para realização do memorial de cálculo das lajes de pontes em balanço, foram adotadas diferentes razões de espessuras da estrutura em balanço, além da variação de posicionamento de aplicação de cargas concentradas sobre o tabuleiro.

A partir dos resultados obtidos foram apresentadas as análises, discussões comparativas inferidas, por fim a conclusão do estudo.

\section{Fluxograma 1.}

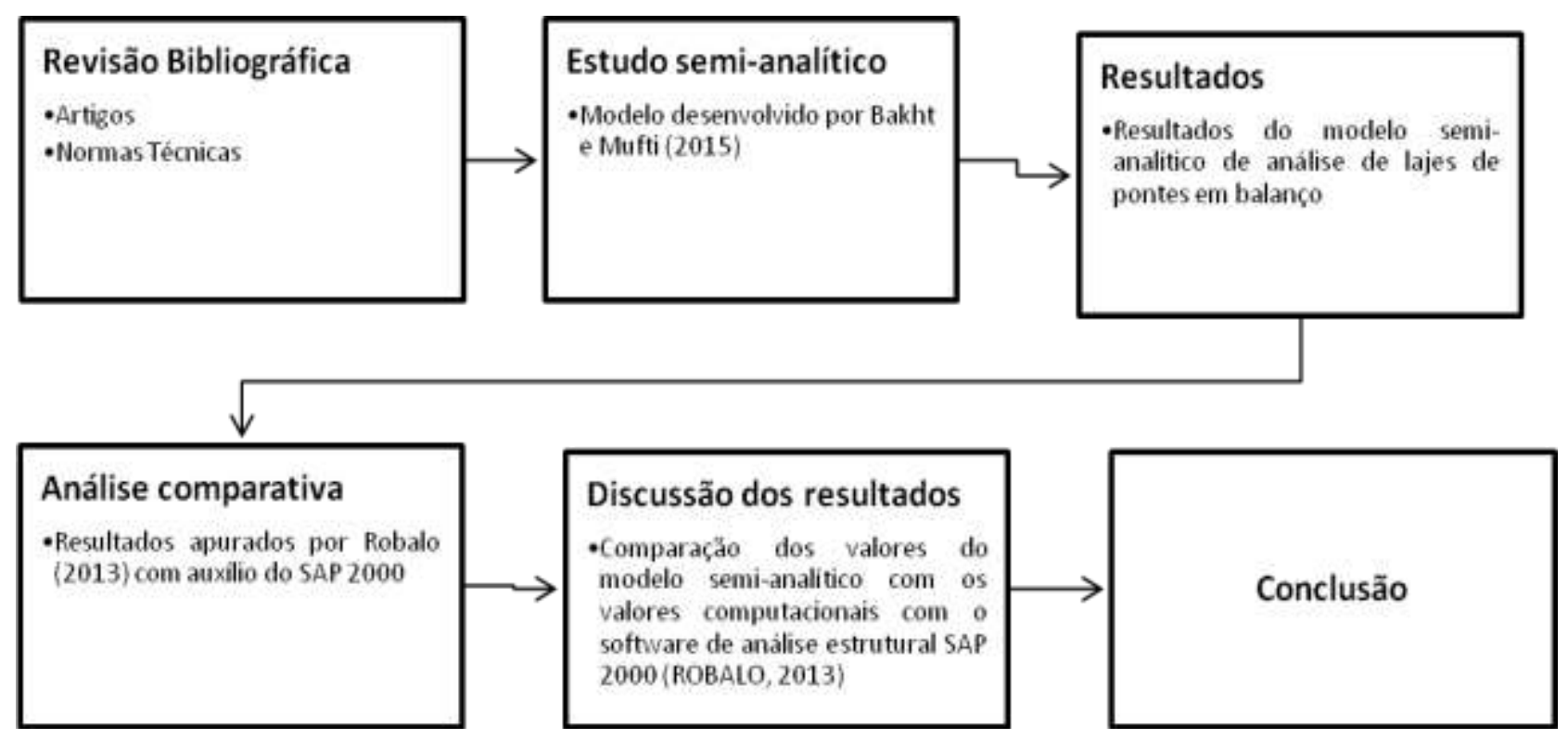

Fonte: Autores.

\section{Referencial Teórico}

\subsection{Histórico}

O processo de construção de pontes faz parte de toda a história de evolução do homem, com os povos primitivos, na elaboração de pontes de cordas, pedras, madeira, na forma de vigas escoradas e vigas armadas simples (Leonhardt, 1979).

$\mathrm{Na}$ história antiga é verificada a presença de construção de pontes associada a características artísticas e até na atualidade tais características permanecem, por isso, também são denominadas obras de artes especiais. Na civilização romana 
é perceptível o legado de tais características, principalmente nos famosos aquedutos que possuíam a função de canalização de água, além da função de transposição e superação dos obstáculos naturais.

Com o passar dos séculos, desenvolvimento dos métodos construtivos e surgimento de novos materiais, as construções das pontes adquiriram novos modelos, formas e tendências, tornando-se não somente obras estruturais, mas também sinônimos de beleza e arte, marcando verdadeiros cartões postais onde são instaladas.

Leonhart (1979) destaca que o surgimento do concreto protendido no século XX impulsionou o mercado da construção civil, com amplo impacto positivo nos projetos de construção de pontes, devido às vigas protendidas que proporcionaram a capacidade e eficiência de vencer grandes vãos a serem transpostos pelo homem.

\subsection{Elementos constituintes da ponte}

As pontes podem ser divididas basicamente em três partes principais de acordo com sua funcionalidade: superestrutura, mesoestrutura e infraestrutura (Pfeil, 1979). Na Figura 1 é apresentado em exemplo de ponte, juntamente com as nomenclaturas dos elementos constituintes da estrutura e suas funcionalidades.

Marchetti (2018) conceitua superestrutura como o elemento de suporte imediato do estrado, é a parte da obra responsável por receber o carregamento do tráfego de veículos, cargas e pessoas, é formada pelas lajes, vigas principais (longarinas), transversinas, cortinas, chanfros, consoles, dentes etc.

Mesoestrutura é a parte estrutural responsável por receber os esforços oriundos da superestrutura (peso próprio e cargas de tráfego) e transmiti-los a infraestrutura através do topo do pilar, é composta pelos aparelhos de apoios, pilares e vigas de contraventamento de pilares. Infraestrutura é a parte estrutural da ponte com a funcionalidade de transmitir os esforços recebidos em toda construção e transmiti-los para o terreno de implantação da obra, rocha ou solo, através dos blocos, estacas, sapatas, tubulões, estaqueamentos, caixões, além das peças de ligações dos seus diversos elementos. (Mendes, 2017).

Figura 1. Elementos constituintes de uma ponte.

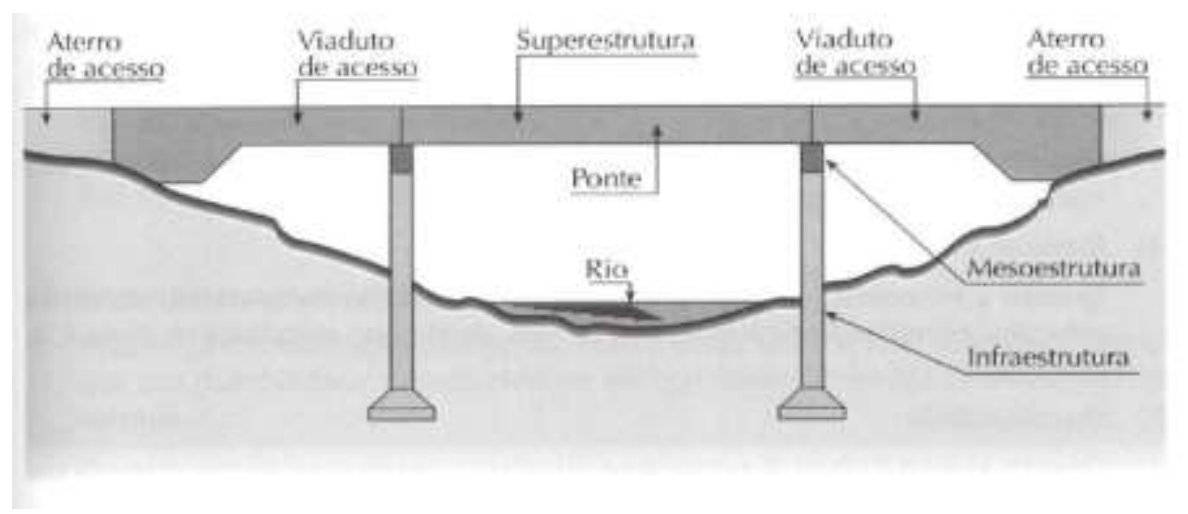

Fonte: Marchetti (2018).

\section{Lajes de Pontes}

As lajes de pontes são elementos estruturais que compõem a superestrutura das obras de arte especiais (OAE's) e possuem a função de suporte das cargas verticais provenientes do peso e locomoção dos veículos, para que possam transmitilas aos outros componentes estruturais até que sejam dissipadas no solo (Pfeil, 1979).

A funcionalidade das lajes de pontes e viadutos se assemelha às lajes utilizadas em edifícios no geral, possuindo como diferença o tipo de carregamento, pois o carregamento predominante é de carga concentrada, representando a aplicação dos eixos dos veículos ou cargas distribuídas em determinadas áreas, além da ação de cargas móveis que se relacionam com o 
efeito da dinâmica. Essas cargas devem ser consideradas no processo de dimensionamento, juntamente com os coeficientes de impacto que recomendam as normas brasileiras e internacionais (Cunha \& Souza, 1994).

Segundo Pfeil (1979) as pontes podem ser classificadas quanto à disposição da seção transversal das vigas ou do sistema estrutural de cada viga. A escolha do sistema de seção transversal, no que diz respeito a tabuleiros de pontes é influenciada por diversos fatores como aponta Leonhardt (1979):

- $\quad$ Processos construtivos, equipamentos e meios disponíveis para execução;

- $\quad$ Tamanho do vão referido ao sistema estrutural proposto;

- A relação entres as cargas atuantes q : g (móvel e peso próprio). Valores elevados de cargas atuantes influenciam em vigas protendidas, com quantidades adicionais de concreto no banzo tracionado, na escolha de seções de duplo T ou em caixão;

- $\quad$ Economia no que diz respeito à escolha do processo construtivo, uma vez que estruturas mais esbeltas exigem um consumo maior de aço do que as menos esbeltas, porém, deve considerar a influência da escolha nas rampas de acesso também;

- $\quad$ Esbeltez desejada ou altura estrutural disponível.

\subsection{Análise de lajes em balanço (cantilever)}

Reissen (2016) conceitua que a variação de espessuras de lajes de pontes (cantilever) apresenta grandes benefícios para estrutura, desde a melhora do efeito estético do elemento estrutural, até a redução do peso próprio da construção e consequentemente redução do peso global e custos com a fundação, além de proporcionar a utilização mais eficiente da armadura no concreto.

As estruturas de lajes de ponte em balanço (cantilever) geralmente são projetadas em forma cônica, possuindo variação de espessura, onde a seção diminui a partir da região engastada (raiz do balanço) até a borda livre ou enrijecida com viga de bordo (Bakht \& Jaeger, 1985).

Para maior eficiência e esclarecimento do estudo faz-se necessária à explicação de algumas terminologias usadas no processo de análise de lajes em balanço. Bakht e Mufti (2015) apresentam tais definições e ilustram de forma didática com a ajuda da Figura 2:

- Raiz do balanço (cantilever) ou Root - É o suporte do balanço, o qual apresenta restrição da rotação e da deflexão vertical, onde podem ser individualmente infinitas ou finitas, ou seja, rígidas ou semirrígidas;

- Direções - As direções paralelas e perpendiculares à raiz são respectivamente consideradas longitudinais e transversais, conforme ilustrado em Figura 2. No que diz respeito aos eixos x e y, estes são paralelos às direções longitudinais e transversais;

- $\quad$ Bordas livres - As bordas livres de uma laje em balanço nas direções paralelas e perpendiculares são definidas como longitudinais e transversais, respectivamente;

- Vão da laje em balanço (cantilever) - Corresponde à distância transversal entre a borda longitudinal e a raiz do balanço. Conforme é ilustrado na Figura 2 este intervalo é denotado como $S_{c}$. Determinados códigos de normas de projeto recomendam que a raiz do balanço seja obtida na borda externa da viga, se for de concreto. Essa recomendação ocorre apenas se a laje em balanço (cantilever) for totalmente fixada com restrição à rotação na sua raiz.

- Intensidades de momento e cisalhamento - As intensidades de momentos e cortantes que agem nas direções $\mathrm{x}$ e y são denotadas como $M_{x}$ e $M_{y}$, respectivamente. Em lajes em balanço, o momento My é mais significativo e definido como intensidade de momento transversal ou intensidade de momento cantilever. Por convenção utilizada nos livros didáticos de 
engenharia, os momentos que agem e causam tensões nas fibras superiores da laje são denominados negativos, enquanto os momentos que geram tensão nas fibras inferiores da laje são determinados como positivos.

Figura 2. Terminologias utilizadas em lajes em balanço.

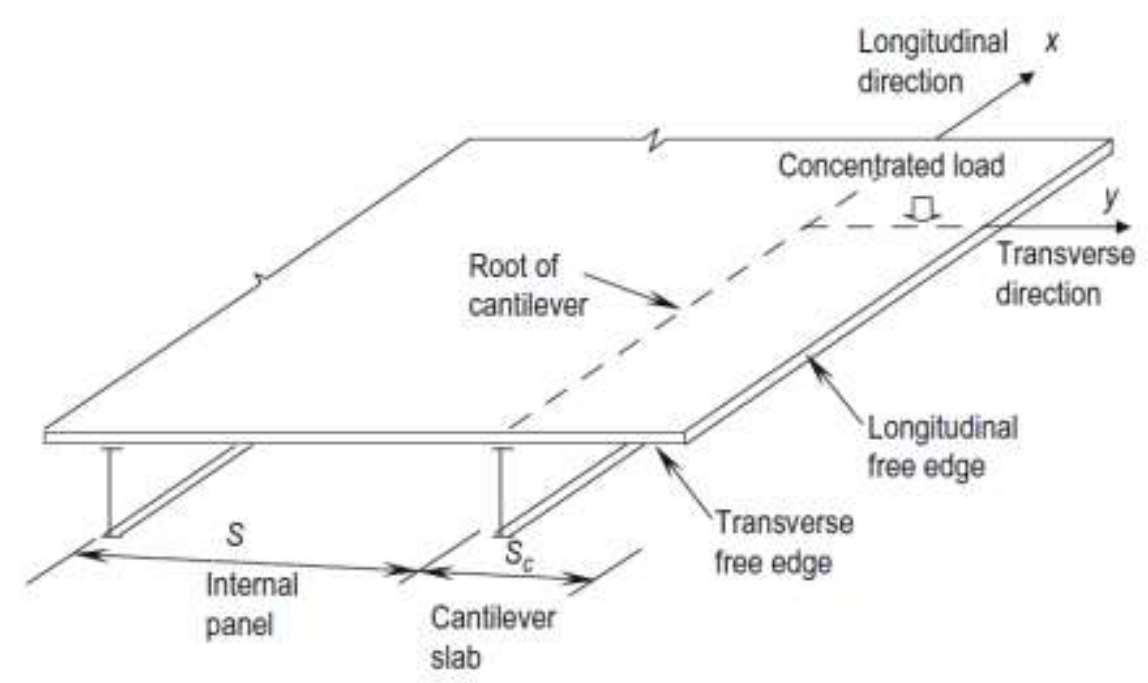

Fonte: Bakht e Mufti (2015).

Os momentos transversais são decorrentes da aplicação de um ou mais carregamentos concentrados nas lajes em balanço de concreto, em lajes com vigas e outros similares tipos de estruturas.

É importante destacar que a nomenclatura da direção da laje em balanço pode causar alguma confusão, onde no caso das lajes em balanço, o termo comprimento (l) geralmente refere-se à distância entre o da raiz do balanço (viga) até a ponta a ponta do bordo livre, representada na Figura 3 a distância 1. Contudo, a nomenclatura de toda ponte, a direção longitudinal é a direção do fluxo do tráfico, paralela às vigas, ou seja, a raiz do balanço.

Figura 3. Exemplos de lajes em balanço.

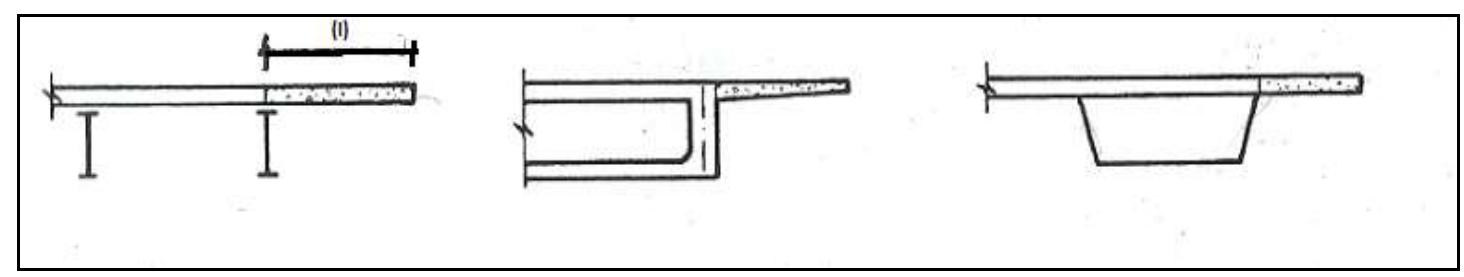

Fonte: Bakht e Jaeger (1985).

A ação de arqueamento de lajes também está presente em lajes em balanço (cantilever), porém, nenhum código de normas no mundo reconhece esse tipo de ação que é muito pouco estudado. A ação de cargas no balanço de uma laje não responde puramente ao comportamento de flexão, mas também se estendem para os painéis internos do tabuleiro, o que deve ser considerado durante a análise da laje de pontes.

O correto estudo e dimensionamento de pontes estão correlacionados a um planejamento e processo de análise eficiente dos fatores que influenciam na intensidade dos momentos de picos transversais que são despertados no elemento 
estrutural, e que comprometem a construção, principalmente com o surgimento de fissuras longitudinais que reduzem a rigidez flexional da laje.

\subsection{Mecânica do comportamento de uma laje em balanço (cantilever)}

Para entendimento, uma correta análise e verificação do comportamento de uma estrutura, bem como a compreensão do funcionamento da construção no que diz respeito à transmissão e fluxo de cargas é essencial para obtenção de segurança no projeto de uma grande estrutura pelo engenheiro que a projeta. É sabido que além da análise estrutural, a verificação dos valores obtidos através programas computacionais estejam obedecendo aos limites esperados (Bakht \& Mufti, 2015). A compreensão do comportamento e funcionamento de susperestruturas de pontes, mais especificamente as lajes, sob a ação de cargas concentradas é primordial para garantia de segurança e eficiência de todo o conjunto estrutural.

Figura 4. Momentos transversais despertados em lajes em balanço.

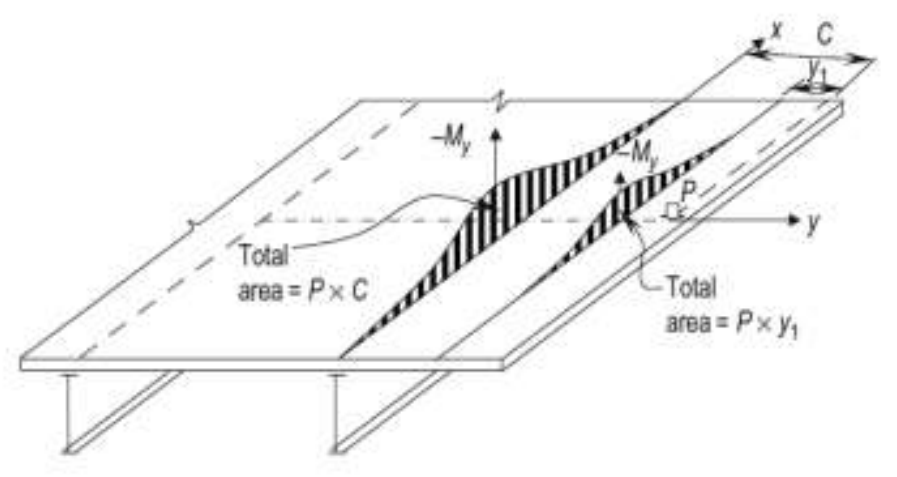

Fonte: Bakht e Mufti (2015)

A Figura 4 auxilia a compreender o exemplo de uma laje de ponte em balanço submetida à ação de uma carga concentrada na parte do bordo livre, apresentando o surgimento de momentos fletores transversais despertados na direção longitudinal do elemento estrutural.

Por observação da Figura 4, pode-se verificar que o momento transversal despertado na raiz do balanço é expresso pelo produto $P C$, onde $P$ corresponde a carga aplicada sobre o tabuleiro e $C$ a distância transversal entre a carga aplicada e a raiz do balanço. O momento fletor transversal de pico é representado por $\mathrm{M}_{\mathrm{y}}$.

A intensidade e distribuição dos momentos fletores $\mathrm{M}_{\mathrm{y}}$ ao longo da seção do balanço da laje é influenciada pelos seguintes fatores:

- $\quad$ Endurecimento da borda;

- Taxa de Espessura da laje - O efeito da relação da taxa de espessura de lajes com a intensidade de momentos devido à distribuição de $\mathrm{M}_{\mathrm{y}}$, pode ser entendida considerando o caso improvável em que a espessura da laje na borda é maior do que na raiz do cantilever (balanço), ou seja $\mathrm{t}_{2} / \mathrm{t}_{1}$ é maior que 1,0 . Pode ser observado que a seção transversal de tal laje é semelhante à seção transversal de uma laje uniforme, com seu bordo enrijecido, ou seja, com a borda reforçada. Com análise comprovada através de estudos, verifica-se que o aumento do valor da razão $\mathrm{t}_{2} / \mathrm{t}_{1}$ ocasiona uma redução do valor de pico de $\mathrm{M}_{\mathrm{y}}$. O encadeamento dedutivo desta observação é que o aumento da intensidade de pico do momento $\mathrm{M}_{\mathrm{y}}$ está relacionado com a redução da relação $\mathrm{t}_{2} / \mathrm{t}_{1}$. Estima-se que o máximo valor de $\mathrm{M}_{\mathrm{y}}$ devido à ação de uma carga concentrada na ponta de uma laje em balanço totalmente fixa com $t_{2} / t_{1}=0,33$, apresenta um aumento de aproximadamente $37 \%$ em relação a uma laje com $\mathrm{t}_{2} / \mathrm{t}_{1}=1,0$. 
- $\quad$ Restrição contra deflexão;

- Restrição contra rotação.

\subsection{Método para uma laje em balanço infinita não enrijecida}

Bakht e Jaeger (1985) observaram que o efeito e os esforços produzidos por uma carga concentrada sobre o balanço tornam-se insignificantes a partir das posições de distância superiores a "2a" no sentido longitudinal do tráfego, onde "a" representa a largura do balanço.

O cálculo para definição do momento transversal $\mathrm{M}_{\mathrm{y}}$ por unidade de comprimento, despertado ao longo da área do balanço, em virtude do carregamento $\mathrm{P}$ de carga concentrada sobre o cantilever é definido por:

$$
\mathrm{M}_{\mathrm{yc}}=\frac{\mathrm{P}}{\pi} \cdot \mathrm{A}^{s} \cdot\left[\frac{1}{\cosh \left(\frac{\mathrm{A}^{y} \cdot \mathrm{x}}{\mathrm{c}-\mathrm{y}}\right)}\right](1)
$$

Onde A' representa um coeficiente que está relacionado com a posição da carga, localização do ponto da raiz do balanço, além da razão de espessura da laje, que relaciona as espessuras do engaste e do bordo livre.

Os valores de A’ são obtidos por meio da consulta aos ábacos apresentados por Bakht e Holland (1976) na Figura 5, onde os valores variam de acordo com as diferentes posições de cargas e pontos de referência na laje em balanço, com variação também das razões de espessuras das lajes de $t_{2} / t_{1}=1,2$ ou 3 , onde $t_{1}$ e $_{2}$ representam as espessuras da laje na raiz e bordo livre respectivamente. Para obtenção de valores não presentes nos gráficos é recomendado a utilização da interpolação linear.

Figura 5. Gráficos para determinação de A' em lajes com balanço não enrijecida.

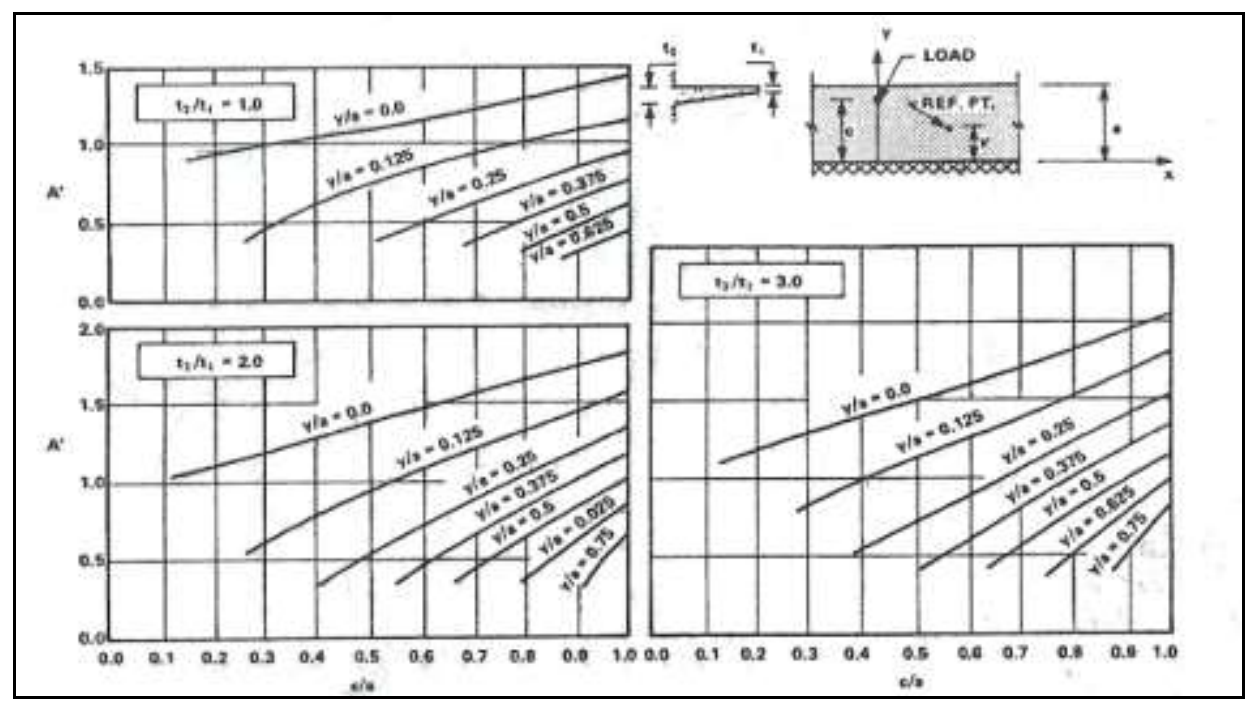

Fonte: Bakht e Jaeger (1985).

São definidos também:

$\mathrm{c}=$ distância do ponto de aplicação da carga ao bordo engastado;

$\mathrm{a}=$ comprimento transversal da laje em balanço ;

$\mathrm{y}=$ ponto de referência onde se deseja determinar o momento fletor $\mathrm{M}_{\mathrm{yc}}$;

$\mathrm{x}=$ distância longitudinal do ponto de referência até o ponto de aplicação da carga $\mathrm{P}$;

$\mathrm{t}_{1}=$ menor espessura da laje em balanço ;

$\mathrm{t}_{2}=$ maior espessura da laje em balanço ; 
O valor máximo do momento $\mathrm{M}_{\mathrm{yc}}$ é despertado na maior distância de aplicação entre o ponto de aplicação da carga e o engaste do balanço.

Bakht e Jaeger (1985) formularam uma equação algébrica onde ocorre a substituição do cosseno hiperbólico (cosh) da Equação 1, passando a possuir a seguinte expressão:

$$
\mathrm{M}_{y}=-\frac{2 \mathrm{P}}{\pi} \cdot B \cdot\left[\frac{(\mathrm{C}-\mathrm{y})^{4}}{\left[(\mathrm{C}-\mathrm{y})^{2}+(\mathrm{Bx})^{2}\right]^{2}}\right]
$$

Onde

$$
\mathrm{B}=\frac{\mathrm{A}^{d}}{2}
$$

As duas equações apresentam praticamente os mesmos resultados. Ambas as equações satisfazem as condições de interação de $\mathrm{M}_{\mathrm{y}}$ em qualquer seção longitudinal de $\mathrm{x}=\infty$ e $\mathrm{x}=-\infty$, é igual o momento negativo total aplicado e My é máximo em $\mathrm{x}=0.0$ e $\mathrm{M}_{\mathrm{y}}=0.0 \mathrm{em} \mathrm{x}=\infty$.

Recomenda-se o uso das equações 1 ou 2 para cálculo de Momentos $\mathrm{M}_{\mathrm{y}}$ na laje em balanço.

Para cálculo do momento no painel interno de lajes de comprimento infinito é apresentado as seguintes equações:

\subsection{Método para uma laje em balanço semi-infinita e não enrijecida}

O método é aplicado para trechos da laje que se situam nas proximidades dos bordos transversais livres. Neste caso, são desconsiderados os trechos infinitos da laje em balanço infinita não enrijecida. A solução apresentada por Bakht e Holland (1976), através das equações 4 e 5 são aplicáveis a lajes em balanço em que a restrição rotacional na raiz é finita. É importante destacar que as variáveis A' e B' são obtidas através das curvas dos ábacos apresentados por meio das Figuras 5 e 7.

Bakht et al. (1979) mostra que o momento $M_{y c}$ despertado na raiz do balanço de uma laje, ou seja, momento no engaste da laje em virtude da carga concentrada perto de uma borda livre transversal é definida por:

$$
\mathrm{M}_{\mathrm{yc}}=\frac{\mathrm{P}}{\pi} \cdot \mathrm{A}^{x} \cdot\left[\frac{1}{\cosh \left(\frac{\mathrm{A}^{x} \cdot \mathrm{x}}{\mathrm{c}}\right)}+\mathrm{B}^{\mathrm{x}} \cdot \mathrm{e}^{-\frac{\mathrm{K} \mathrm{K}_{\mathrm{b}}}{\mathrm{a}}}\right]
$$

Os valores de K são definidos por:

$$
\mathrm{K}=\frac{\mathrm{a}}{\mathrm{c}} \cdot \frac{\mathrm{A}^{\mathrm{s}} \cdot \mathrm{B}^{j}}{2} \cdot\left[\frac{1}{\operatorname{arctg}\left(\mathrm{e}^{-\frac{A^{r} \mathrm{xR}_{\mathrm{z}}}{\mathrm{c}}}\right)}\right]
$$


Figura 6. Ilustração para representação do método de análise de lajes em balanço (cantilever) de comprimento semi-infinito.

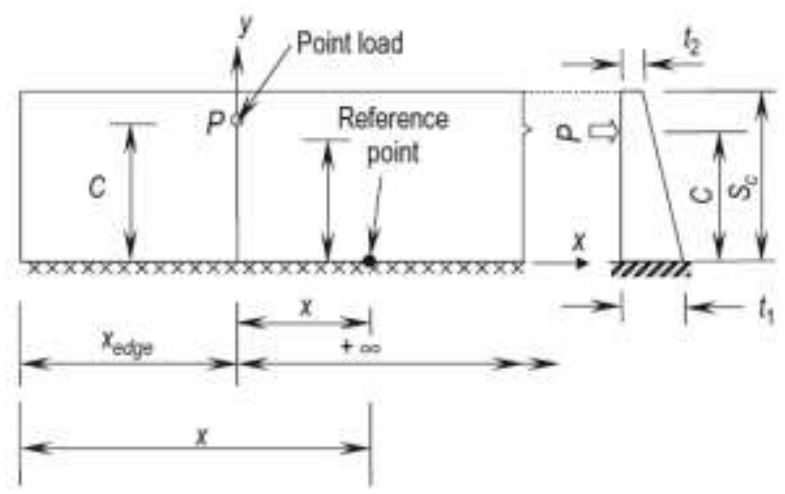

Fonte: Bahkt e Muft (2015)

Os valores de A' são obtidos dos gráficos da Figura 5 e os valores de B' dos gráficos da Figura 7, para as mais variadas relações $\mathrm{t}_{2} / \mathrm{t}_{1}$ e $\mathrm{y} / \mathrm{a}$.

A Figura 6 auxilia na compreensão da aplicação do método, esboçando a seção da laje em balanço, bem como as posições de aplicação de carga em relação à direção longitudinal e transversal da peça, uma vez que as equações propostas utilizam tais variáveis de coordenadas, tomando pontos de referência sobre a parte do tabuleiro em balanço.

Figura 7. Gráficos para determinação de B' para lajes não enrijecidas

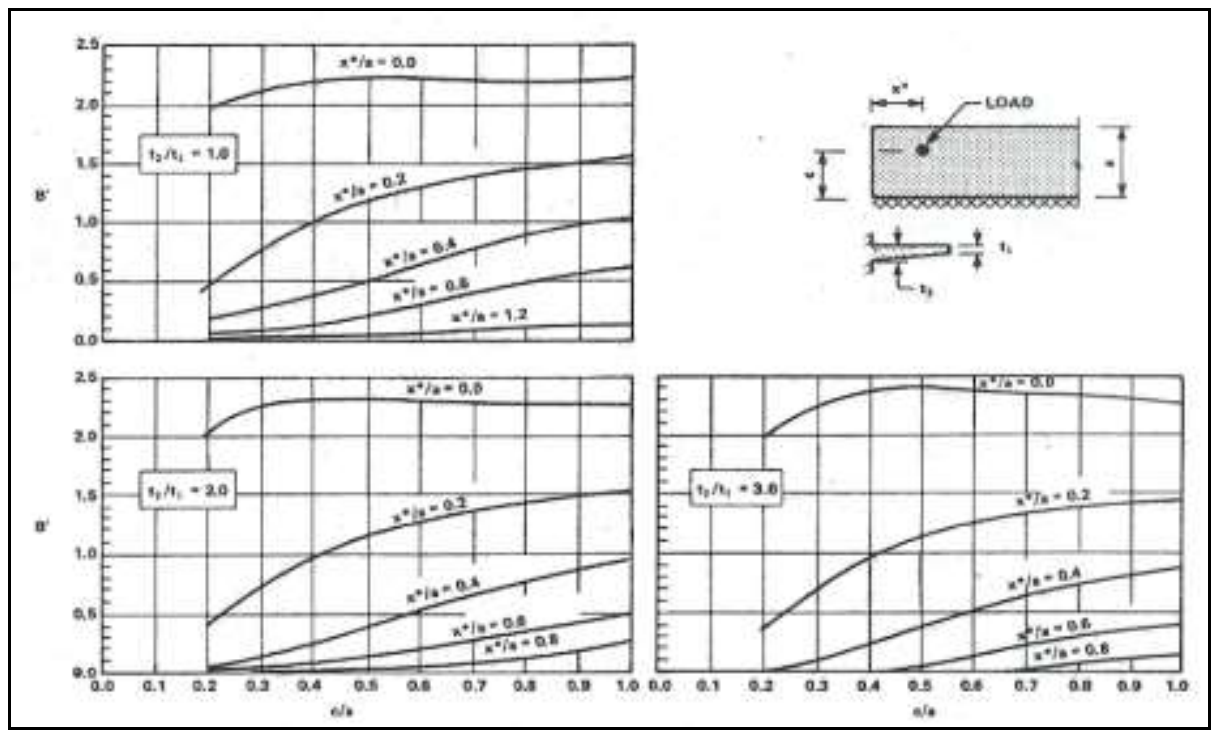

Fonte: Bakht e Jaeger (1985).

\subsection{Método para uma laje em balanço infinita de bordo enrijecido}

As vigas de bordo invertidas utilizadas em lajes em balanço possuem a função de enrijecer o bordo livre, sendo estruturas monolíticas à laje a que estão conectadas. Essas vigas possuem a finalidade de melhorar a distribuição de cargas ao longo da laje em balanço.

O tratamento para essas estruturas é quase similar ao das lajes não enrijecidas, onde a diferença ocorre no parâmetro A’ obtido através do ábaco da Figura 8, variando de acordo com a rigidez flexional da viga de bordo, rigidez flexional da laje em balanço e a razão de espessuras escolhidas. O valor de A' também está associado à razão entre a rigidez à flexão da viga de bordo para a rigidez total da laje em balanço em torno do seu eixo neutro. 
Para cálculo dos momentos transversais no balanço, em virtude de uma carga concentrada é utilizada a Equação 4, onde o coeficiente A' é obtido a partir da consulta ao ábaco da Figura 8. Esse método é aplicado apenas nos casos em que y é menor que $\mathrm{c}$.

Bakht e Jaeger (1985) definiram alguns outros gráficos para cálculo de diferentes valores de A', nas situações em que as lajes em balanço são enrijecidas com reforço estrutural em suas bordas. Para utilização dos valore de A' é necessário a consulta aos gráficos apresentados que associam o momento de inércia da borda da seção transversal da viga $\left(\mathrm{I}_{\mathrm{S}}\right)$ com o momento de inércia da laje em balanço $\left(\mathrm{I}_{\mathrm{b}}\right)$ na seção transversal.

É importante destacar que os novos valores de A' também estão intrínsecos a variação da razão de espessuras da laje em balanço e ao posicionamento da carga (y/a), conforme pode ser verificado na Figura 8.

As Equações 6 e 7 definem as rigidezes flexionais dos elementos estruturais (viga de bordo e laje):

$$
\begin{gathered}
I_{s}=\frac{a}{48} \cdot\left(t_{2}^{a}+t_{2}^{2} \cdot t_{1}+t_{2} \cdot t_{1}^{2}+t_{1}^{a}\right)=\frac{a}{48} \cdot \frac{t_{2}^{4}-t_{1}^{4}}{t_{2}-t_{1}}(6) \\
I_{b}=\frac{b \cdot h^{a}}{12}(7)
\end{gathered}
$$

$\mathrm{I}_{\mathrm{S}}=$ momento de inércia da área da laje em balanço com variação de espessura em relação ao eixo médio da seção transversal;

$\mathrm{I}_{\mathrm{b}}=$ momento de inércia da área da viga de bordo em relação ao eixo médio da seção transversal;

$\mathrm{b}=$ espessura da viga de bordo;

$\mathrm{h}=$ altura da viga de bordo;

$\mathrm{t}_{2}=$ maior espessura da laje em balanço;

$\mathrm{t}_{1}=$ menor espessura da laje em balanço;

Figura 8. Gráficos para determinação de A' para lajes infinitas e enrijecidas.

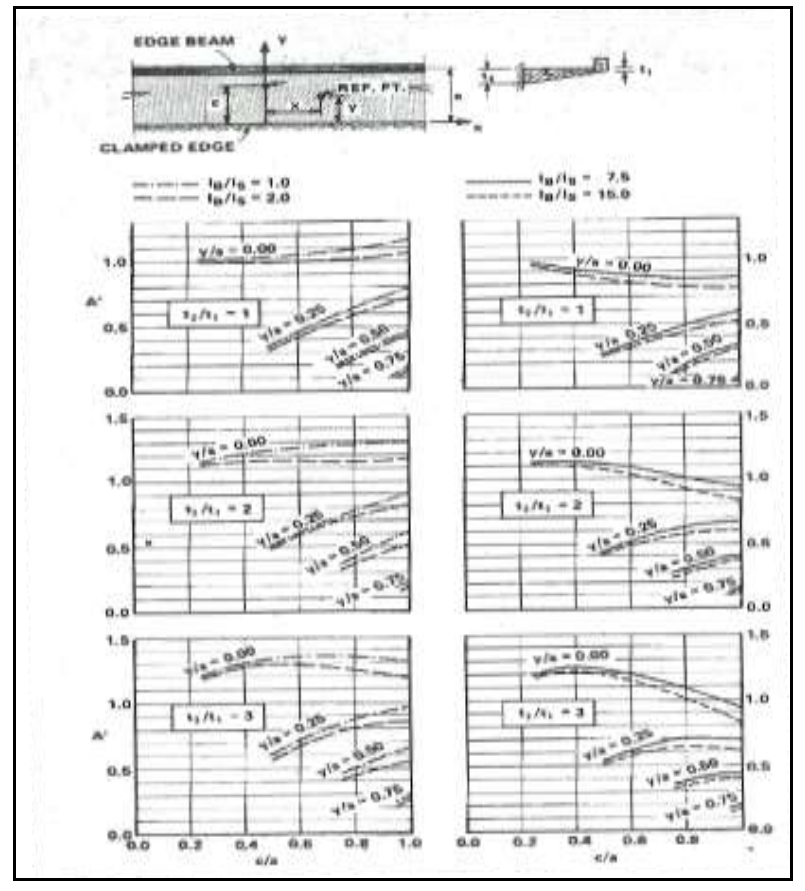

Fonte: Bakht e Holland (1976). 


\section{Estudo de Caso}

\subsection{Dados de Entrada}

Para estudo e comparação dos resultados gerados pelo método semi-analítico desenvolvido por Bakht e os obtidos através do método de elementos finitos (MEF), com auxílio do software SAP 2000, este trabalho utilizou os dados presentes em Robalo (2013).

Para o caso apresentado a razão utilizada entre o vão da laje em balanço (cantilever) e o vão interno do tabuleiro da ponte é $S_{c} / S=0,5$. Os modelos analisados apresentam as seguintes características:

- Vão do balanço da laje de $2 \mathrm{~m}$, sem enrijecimento das bordas;

- $\quad$ Rigidez flexional infinita das vigas e rigidez torcional zero;

- $\quad$ Razão de variação das espessuras das lajes (t2/t1=1,2 e3);

- $\quad$ Posições das cargas concentradas no tabuleiro $\left(\mathrm{c} / \mathrm{S}_{\mathrm{c}}=0,2,0,4,0,6,0,8,1,0\right)$;

- $\quad$ Coeficiente de Poisson $v=0,2$;

- $\quad$ Posição do $\mathrm{x}=1 \mathrm{~m}$.

Para análise de laje em balanço (cantilever) reforçada com viga de bordo, Robalo (2013) utilizou os dados seguintes dados:

- $\quad$ Comprimento transversal do balanço $\mathrm{S}_{\mathrm{c}}=2 \mathrm{~m}$;

- $\quad$ Coeficiente de Poisson $v=0$;

- $\quad$ Na análise modelar foi considerada uma restrição rotacional infinita;

- $\quad$ Espessura constante da laje em balanço $\mathrm{t} 2 / \mathrm{t} 1=1$;

- $\quad$ Viga de bordo com dimensões resultando $\mathrm{I}_{\mathrm{b}} / \mathrm{I}_{\mathrm{s}}=1$;

- $\quad$ Posições de cargas concentradas variando $\left(\mathrm{c} / \mathrm{S}_{\mathrm{c}}=0,2,0,4,0,6,0,8,1,0\right)$ (Figura 10).

\subsection{Análise e Discussões}

A partir dos resultados obtidos por Robalo (2013), verifica-se que os valores alcançados através do método semianalítico criado por Bakht e Mufti (2015) podem ser significativamente diferentes dos valores fornecidos através do SAP2000, quando a carga aplicada se encontra próxima a viga longitudinal, ou seja, próximo à raiz do balanço, conforme apresentado nos gráficos e a razão entre as espessuras é menor.

Em relação aos momentos transversais máximos, é perceptível que ambos os métodos de análise possuem variações pequenas, quando comparados entre si. 
Figura 9. Resultados apurados através do SAP2000.

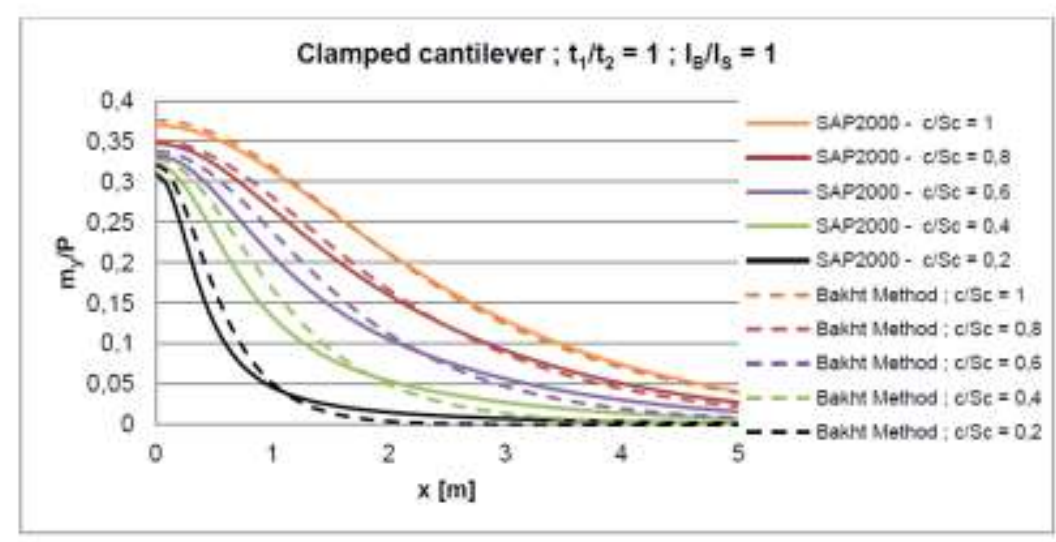

Fonte: Robalo (2013).

Com base nos resultados esboçados no gráfico da Figura 9, observa-se que o método estabelecido fornece resultados muito bons para os casos de lajes em balanço com borda enrijecida com vigas de bordo (sem consideração da influência da rigidez da laje interna), embora haja algumas pequenas diferenças quando a carga está locada próxima da borda fixada do balanço.

É importante destacar que em sua análise, Robalo (2013) constatou que os valores obtidos levando em consideração a flexibilidade do painel interno da laje da ponte e o caso de lajes em balanço totalmente fixas apresentam diferenças significativas, ao se considerar a restrição rotacional finita.

Tabela 1. Comparativo estudo analítico.

\begin{tabular}{ccccccc}
\hline \multicolumn{7}{c}{ Comparativo estudo analítico } \\
\hline Posições da carga & $\begin{array}{c}\text { P=1 } \\
\text { KN }\end{array}$ & $\begin{array}{c}\text { ROBALO } \\
\text { SAP2000 }\end{array}$ & $\begin{array}{c}\text { P=1KN } \\
\text { t2/t1=2 }\end{array}$ & $\begin{array}{c}\text { ROBALO } \\
\text { SAP2000 }\end{array}$ & $\begin{array}{c}\text { P=1KN } \\
\text { t2/t1=3 }\end{array}$ & $\begin{array}{c}\text { ROBALO } \\
\text { SAP2000 }\end{array}$ \\
$\mathbf{0 , 1}$ & $-0,008$ & & $-0,004$ & & $-0,004$ & \\
$\mathbf{0 , 2}$ & $-0,058$ & $-0,05$ & $-0,045$ & $-0,05$ & $-0,038$ & $-0,05$ \\
$\mathbf{0 , 3}$ & $-0,116$ & & $-0,102$ & & $-0,094$ & \\
$\mathbf{0 , 4}$ & $-0,167$ & $-0,15$ & $-0,157$ & $-0,14$ & $-0,150$ & $-0,14$ \\
$\mathbf{0 , 5}$ & $-0,210$ & & $-0,207$ & & $-0,203$ & \\
$\mathbf{0 , 6}$ & $-0,248$ & $-0,24$ & $-0,253$ & $-0,22$ & $-0,251$ & $-0,22$ \\
$\mathbf{0 , 7}$ & $-0,279$ & & $-0,294$ & & $-0,295$ & \\
$\mathbf{0 , 8}$ & $-0,307$ & $-0,29$ & $-0,332$ & $-0,34$ & $-0,337$ & $-0,30$ \\
$\mathbf{0 , 9}$ & $-0,338$ & & $-0,369$ & & $-0,377$ & \\
$\mathbf{1}$ & $-0,362$ & $-0,34$ & $-0,400$ & $-0,42$ & $-0,415$ & $-0,39$ \\
\hline
\end{tabular}

Fonte: Autores.

A Tabela 1 apresenta os valores comparativos obtidos através do método semi-analítico e dos resultados alcançados por Robalo (2013) com a utilização do software baseado no método de elementos finitos. É verificado ainda que a intensidade dos momentos fletores transversais está associada a razão das espessuras das lajes, onde é inferido que a intensidade de picos é diretamente proporcional a razão das espessuras. Porém, com a inserção de vigas de bordo nas lajes em balanço, foi constatada a redução dos momentos transversais, devido a maior distribuição da carga concentrada sobre o tabuleiro e essa eficiência é aumentada quando associada à variação de razão de espessuras, conforme foi verificado por Correia e Mendes (2021). 
Figura 10. Resultados apurados para $\mathrm{t} 1 / \mathrm{t} 2=1$

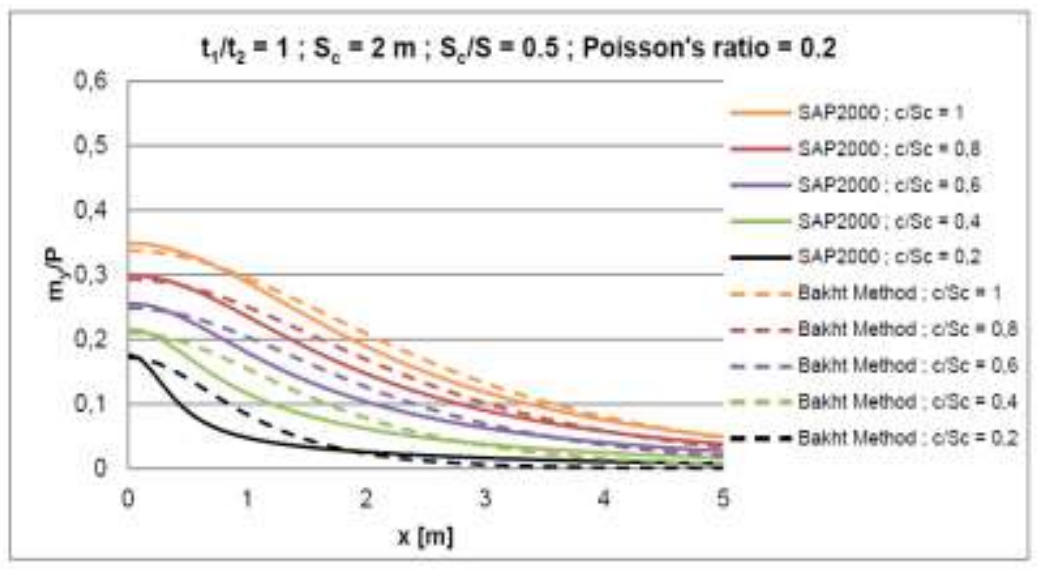

Fonte: Robalo (2013).

Figura 11. Resultados apurados para $\mathrm{t} 1 / \mathrm{t} 2=2$.

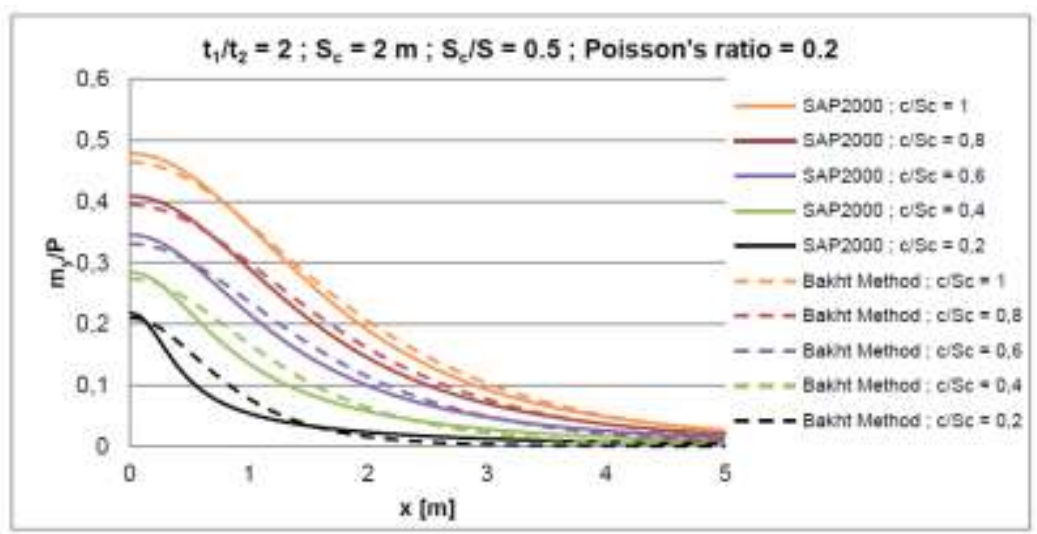

Fonte: Kobaı ( $\angle \mathrm{U} 13)$.

Figura 12. Resultados apurados para $t 1 / t 2=3$

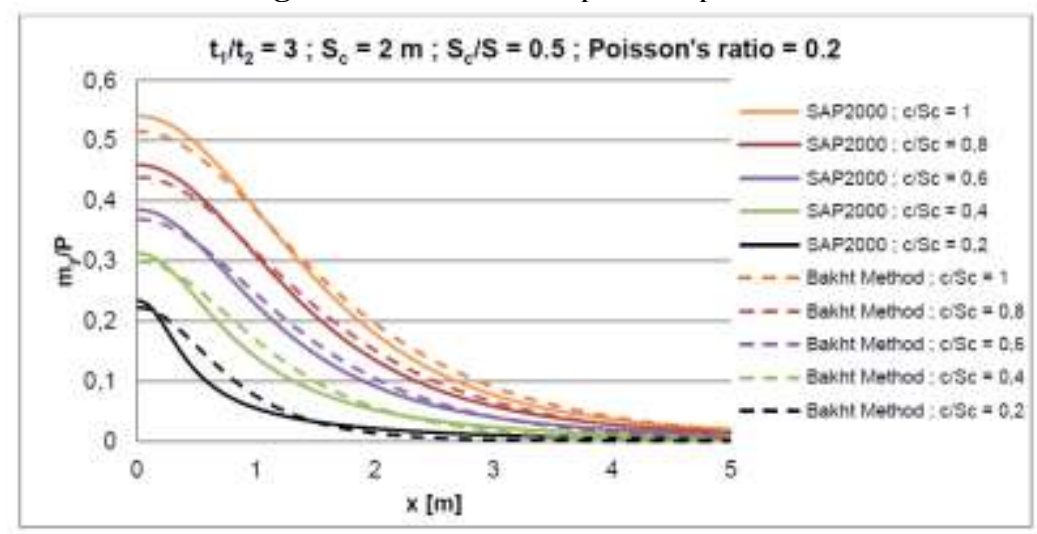

Fonte: Robalo (2013).

Através do estudo comparativo realizado neste trabalho, verificaram-se algumas pequenas diferenças de precisão nos valores obtidos através do modelo matemático desenvolvido por Bakht e Mufti (2015) e os valores alcançados com o apoio do software SAP 2000 por Robalo (2013). Essas diferenças podem ser entendidas como precisão de análise, uma vez que o programa computacional é pautado pelo MEF (método de elementos finitos), enquanto o modelo analítico é baseado na análise matemática, o que gera uma maior precisão de estudo da estrutura. 
Porém, não obstante dos valores obtidos através do software, pode-se constatar por análise da tabela que os resultados alcançados com o método semi-analítico exposto são muito próximos, apresentando pouca variação e isso é perceptível ao observar os gráficos das Figuras 10, 11 e 12 elaborados por Robalo (2013), os quais ilustram as curvas comparativas usando as duas formas de análise.

A partir dos resultados obtidos podemos inferir por análise também que o comportamento dos momentos fletores despertados na direção transversal tende a reduzir nos pontos distantes da posição de aplicação da carga concentrada. Isso foi observado por Bakht e Jaeger (1985), ao afirmar que os valores dos momentos transversais tendem a se aproximar de zero, à medida que a distância do local de análise ultrapassa o dobro do comprimento transversal da laje em balanço (cantilever).

\section{Conclusão}

Conclui-se por análise dos resultados desse estudo que a magnitude dos momentos de picos transversais tende a zero nos locais onde o ponto de análise ultrapassa o dobro do comprimento do balanço

Cumpre ressaltar que a intensidade dos momentos fletores transversais despertados na direção longitudinal do bordo engastado está associada à razão de espessuras utilizadas nas lajes, onde é perceptível que a razão de espessuras é diretamente proporcional à intensidade dos momentos fletores que surgem na peça.

A propagação dos momentos fletores transversais despertados está associada também às posições de aplicação de carga concentrada na estrutura, de forma que ocorre o aumento dos momentos fletores à medida que a caga concentrada se distancia da raiz do balanço.

No estudo comparativo com os resultados observados por Robalo (2013), foi inferido que os valores fornecidos com auxílio do software SAP2000 são bem próximos aos valores calculados manualmente através do modelo matemático desenvolvido por Bakht e Jaeger (1985), porém, em determinadas posições constata-se uma pequena variação de valores devido a precisão do método aplicado, quando comparado com o software que utiliza o MEF (método de elementos finitos), juntamente com as pequenas diferenças ao se levar em consideração as normas estrangeiras, nas quais o software SAP 2000 é baseado, quando comparado a norma brasileira.

Neste estudo foi analisado o comportamento de lajes de pontes em balanço, e com base em todos os resultados, verificações e comparações realizadas é possível afirmar que a utilização de vigas de bordo, bem como a associação de variação da razão de espessuras do cantilever ocasionam uma melhor eficiência na redução dos momentos transversais despertados no balanço do tabuleiro, e com isso a redução do surgimento de fissuras longitudinais que reduzem a rigidez flexional da estrutura.

Por fim, como proposta de trabalhos e pesquisas futuras, recomenda-se a elaboração de uma análise comparativa do modelo matemático estudado nesse trabalho, com os resultados apurados pelo software de análise estrutural ANDECAS, baseado no método analítico, com a finalidade de comparação da precisão de resultados de cada modelo com aplicação de cargas em conjunto, simulando as ações reais de tráfegos de vários veículos sobre o tabuleiro.

\section{Referências}

Associação Brasileira de Normas Técnicas. ABNT NBR 7188 (2013). Carga móvel rodoviária e de pedestres em pontes, viadutos, passarelas e outras estruturas.

Associação Brasileira de Normas Técnicas, ABNT NBR 7187 (2003). Projeto de pontes de concreto armado e de concreto protendido.

Bakht, B. \& Mufti, A. (2015). Bridges analysis, design, structural health monitoring, and rehabilitation. Springer.

Bakht, B. \& Jaeger, L. G. (1985). Bridge analysis simplified, New York. McGraw-Hill Book Company.

Bakht B. \& Holland, D. A. (1976) A manual method for the elastic analysis of wide cantilever slabs of linearly varying thickness. Can J Civ Eng 3(4):523-530 
Research, Society and Development, v. 10, n. 15, e20101521141, 2021

(CC BY 4.0) | ISSN 2525-3409 | DOI: http://dx.doi.org/10.33448/rsd-v10i15.21141

Bakht. B., Aziz, T.S. \& Bantusevicius, K.F. (1979). Manual analysis of cantilever slabs of semi-infinite width. Can J Civ Eng 6(2):227-231

Correia, L. B.\& Mendes, L. C. (2021). Analysis of thickness in deck slab overhangs. Research, Society and Development, 10(8), e50610817556: $10.33448 /$ rsd-v10i8.17556.

Cunha, A. J. P.\& Souza, V. C. M. (1994). Lajes em concreto armado e protendido. EDUFF.

Cusens, A. R. \& Pama, R. P (1975). Bridge deck analysis. John Wiley \& Sons.

El Debs, M. K., Malite, M., Takeya, T., Munair Neto, J.\& Hanai, J.B. (2001). Análise das consequências do Tráfego de combinações de veículos de carga (cvcs) sobre as Pontes da rede viária sob jurisdição do DER-SP. Departamento de Estradas de Rodagem do Estado de São Paulo, Relatório Técnico, Minerva.

Leonhardt, F. (1982). Brucken-Bridges. The Architetural Press.

Meireles, A. P. C. Levantamento e Diagnóstico de uma ponte metálica antiga. 2010. 74p. Dissertação de Mestrado (Mestrado em Engenharia Civil) Departamento de Engenharia Civil, Faculdade de Engenharia da Universdade do Porto, Porto - Portugal.

Mendes, L. C. (2017). Pontes. Editora da Universidade Federal Fluminense.

Marchetti, O. (2018). Pontes de concreto armado. Blucher.

Munoz, L. J. V. (2020). Bridge Overhangs Slabs with Edge Beams. 125p. Doctoral Thesis (Doctoral Thesis in Structural Engineering and Bridges) - KTH Royal Institute of Technology, KTH, Stockholm.

O’Connor, Daniel S. (1991) - La gestion de puentes en Estados Unidos - Simpósio Nacional sobre conservacion, rehabilitacion y gestion de puentes, Madrid.

O’Connor, C. (1976). Pontes-superestruturas. Livros Técnicos e Científicos.

Pfeil, W. (1979). Pontes em Concreto Armado. Livros Técnicos e Científicos Editora S/A.

Pfeil, W. (1983). Pontes: Curso Básico - Editora Campus Ltda.

Quiroga, A. F. S. (1983). Cálculo de Estructuras de Puentes de Hormigon. Editorial Rueda.

Reissen, K. \& Hegger, J. (2015). Experimental investigations on the shear capacity of RC cantilever bridge deck slabs under concentrated loads - Influences of moment-shear ratio and an inclined compression zone. 16th European Bridge Conference, Edinburgh, Scotland

Rowe, R. E. (1972). Concrete bridge design. Science Publishers LTD.

Silva, S. C. (2016). Análise paramétrica de superestrutura de ponte em viga contínua. 315p. Trabalho de Conclusão de curso (Graduação em Engenharia Civil) - Escola Politécnica, UFRJ, Rio de Janeiro.

Tardivo, F. G. (2014). Estudo de Esquemas estruturais e modelagem de tabuleiros de pontes esconsas. 162p. Dissertação de mestrado (Mestrado em Engenharia Civil) - Escola Politécnica da Universidade de São Paulo, USP, São Paulo.

Vitório, A. (2002). Pontes Rodoviárias. CREA-PE. 